te wurden die Zahl der Tage mit Migräne, die durchschnittliche Schmerzintensität und die medikamentöse Analgesie gewählt. Dokumentiert wurde alle vier Wochen über einen Zeitraum von insgesamt 24 Wochen.

Für die Elektroakupunktur, die von Ärzten mit mindestens vierjähriger klinischer Erfahrung mit dieser Methode beidseitig abwechselnd angewandt wurde, verwendeten die Mediziner das HANS $^{\circledR}$-System des Han-Instituts in Nanjing. Die Stimulationsfrequenz betrug 2/100 Hz mit einem Wechsel alle drei Sekunden. Die Intensität variierte zwischen 0,1 und 1,0 mA, je nach Präferenz des Patienten. Pro Sitzung wurden vier Akupunkturpunkte verwendet. Dazu gehörten auf jeden Fall die zwei Punkte GB20 (im Nacken auf der Haargrenze in Höhe des Ohrläppchens) und GB8 (oberhalb des äußeren Ohres). Zur Auswahl standen zudem die Punkte
SJ5, GB34, BL60, SI3, LI4, ST44, LR3 und GB40. In der Kontrollgruppe wurden Punkte gewählt, die nicht als klassische Akupunkturpunkte gelten. Anhand des „De Qi“-Gefühls, das die Patienten bei der Behandlung beschrieben, beurteilten die Ärzte, ob die Akupunktur jeweils richtig durchgeführt wurde.

Wie Zhao und ihre Kollegen berichten, hatte die Häufigkeit der Migräneattacken in der Gruppe mit korrekter Akupunktur um 3,2 abgenommen, in der Gruppe mit Scheinakupunktur dagegen nur um 2,1. Zwischen der Gruppe mit Scheinakupunktur und den $\mathrm{Pa}$ tienten ohne Intervention gab es bei diesem Parameter keinen signifikanten Unterschied. Die durchschnittliche Zahl der Tage mit Migräne war in der Verumgruppe ebenfalls niedriger als in der Gruppe mit Scheinakupunktur; dieser Unterschied war signifikant (2,0 vs. 3,$1 ; p=0,005)$. Schließlich war auch die Kopfschmerzintensität, beurteilt mithilfe der visuellen Analogskala (VAS-Score), mit korrekter Akupunktur signifikant niedriger (3,4 vs. 4,$2 ; \mathrm{p}=$ 0,008). Der Bedarf an Schmerzmitteln unterschied sich jedoch nicht.

Die noch bis Herbst 2017 gültige Leitlinie der Deutschen Gesellschaft für Neurologie kommt zu dem Schluss, dass Akupunktur in der Prophylaxe der Migräne wirksam ist. Allerdings sei eine Scheinakupunktur genauso wirksam wie eine Akupunktur nach den TCM-Prinzipien. Auf die Wirksamkeit einer Elektroakupunktur wird in der Leitlinie nicht eingegangen.

\section{Quelle}

Zhao L et al. The Long-term Effect of Acupuncture for Migraine Prophylaxis. A Randomized Clinical Trial. JAMA Int Med 2017; online 20. Februar

\title{
Tumorschmerz: Bei jedem Fünften neuropathisch
}

\section{Unter ambulant versorgten Krebspatienten hat fast jeder dritte chronische Tumorschmerzen. Bei jedem fünften Schmerzpatien- ten handelt es sich um neuropathische Schmerzen mit verstärk- ter Intensität, wie aus einer französischen Studie hervorgeht.}

» Bisherige Schätzungen zur Häufigkeit neuropathischer Schmerzen bei Patienten mit chronischen Tumorschmerzen beruhen auf Studien mit stationär behandelten Patienten in speziellen Einrichtungen wie Schmerz- und Palliativkliniken. Nach Ansicht von Dr. Didier Bouhassira, Gesundheitsinstitut INSERM in Boulogne-Billancourt/ Frankreich und seinen Kollegen sind Untersuchungen ambulanter Tumorschmerzpatienten repräsentativer.

Deshalb haben sie den Anteil der Krebspatienten mit chronischen Schmerzen mit oder ohne neuropathische Schmerzen ermittelt, die in einem Zeitraum von zwei Wochen die ambulante Abteilung einer Spezialklinik aufsuchten. Als chronisch waren Schmerzen definiert, wenn sie mindestens drei Monate anhielten. Neuropathische Schmerzen wurden mithilfe des DN4Scores (Douleur Neuropathique en 4 Questions) ermittelt. Zudem bestimmten die Ärzte prospektiv die Inzidenz chronischer Schmerzen mit oder ohne neuropathische Schmerzen.

Eingebunden waren insgesamt zwölf Einrichtungen unter 20 Krebszentren und 25 onkologischen Abteilungen (1.805 Patienten). Patienten, die zum Zeitpunkt der ersten Untersuchung keine Schmerzen hatten, wurden zur Ermittlung der Inzidenz nach drei und sechs Monaten erneut untersucht. Der Auswertung zufolge hatten 28,2\% der Studienteilnehmer chronische Schmerzen. Bei 47,6\% der Patienten vermuteten die Ärzte den Tumor als Ursache, bei 23,7\% die Krebstherapie und bei 23,5\% eine tumorunabhängige Ursache. Fast jeder zweite Patient klagte über Schmerzen im Rücken, 45,6\% über Schmerzen in Hals und Schultern, $43,6 \%$ in den Beinen und $36,6 \%$ in den Armen. Die Prävalenz neuropathischer Schmerzen lag bei 20,9\% ( $n=106)$ der Patienten mit chronischen Schmerzen (5,9\% aller Patienten). Das stimmte bei $88 \%$ der Patienten mit der klinischen
Beurteilung überein. Bei 86 Patienten standen die neuropathischen Schmerzen im Zusammenhang mit dem Tumor. Nach Ansicht der Ärzte beruhten die neuropathischen Schmerzen bei fast $48 \%$ der Patienten auf einer lokalen Progression der Tumoren oder auf $\mathrm{Me}$ tastasen, bei mehr als $45 \%$ auf einer oder mehreren Krebstherapien und bei $7 \%$ auf beidem. Die Intensität der neuropathischen Schmerzen sei dabei höher gewesen.

Für die Ermittlung der Schmerzinzidenz standen die Befunde von 1.285 Studienteilnehmern zur Verfügung. Bei 873 erfolgte mindestens eine Untersuchung drei oder sechs Monate nach der ersten. 599 Patienten wurden zu beiden Zeitpunkten untersucht. Die Inzidenz chronischer Tumorschmerzen lag in diesem Zeitraum zwischen 13 und $28 \%$. Als neuropathisch wurden Schmerzen bei knapp 20\% dieser Patienten eingestuft.

$(P L)$

\section{Quelle}

Bouhassira D et al. Prevalence and incidence of chronic pain with or without neuropathic characteristics in patients with cancer. PAIN 2017; online 4. März 